\title{
FIXED POINT THEOREMS FOR GENERALIZED NONEXPANSIVE MAPPINGS
}

\author{
CHI SONG WONG
}

(Received 12 July 1972, revised 25 August 1972)

Communicated by E. Strzelecki

\section{Introduction}

Let $S, T$ be self-mappings on a (non-empty) complete metric space $(X, d)$. Let $a_{i}, i=1,2, \cdots, 5$, be non-negative real numbers such that $\sum_{i=1}^{5} a_{i}<1$ and for any $x, y$ in $X$,

$$
\begin{aligned}
d(S(x), T(y)) \leqq & a_{1} d(x, y)+a_{2} d(x, T(y))+a_{3} d(y, S(x)) \\
& +a_{4} d(x, S(x))+a_{5} d(y, T(y)) .
\end{aligned}
$$

The Banach contraction mapping theorem says that $T$ has a unique fixed point if $S=T$ and $a_{2}=a_{3}=a_{4}=a_{5}=0$. Kannan [15] proved that $T$ has a unique fixed point if $S=T$ and $a_{1}=a_{2}=a_{3}=0$. Reich [26] proved that $T$ has a unique fixed point if $S=T$ and $a_{2}=a_{3}=0$. Hardy and Rogers [13] proved that $T$ has a unique fixed point if $S=T$. Gupta and Srivastava [27] proved that $S, T$ have a unique common fixed point if $a_{1}=a_{2}=a_{3}=0$ and $a_{4}=a_{5}$. We proved [30] that $S, T$ have a unique common fixed point if $a_{2}=a_{3}$ and $a_{4}=a_{5}$. When $S=T$, because of the symmetry in $x, y$, one can, without loss of generality, assume that $a_{2}=a_{3}$ and $a_{4}=a_{5}$. So our result generalizes all of the results mentioned above. In general, there is however no such symmetry as $a_{2}=a_{3}$ and $a_{4}=a_{5}$. There are examples [30] of $S, T$ which satisfy the above conditions, but (1) does not hold for any $a_{i}, i=1,2, \cdots, 5$, in $[0,1]$ with $a_{2}=a_{3}, a_{4}=a_{5}$ and $\sum_{i=1}^{5} a_{i}<1$. By extending the idea of Rakotch [25], we [30] introduced monotonically non-increasing self-mappings $\alpha_{i}, i=1,2, \cdots, 5$, on $[0, \infty]$ such that $\alpha_{2}=\alpha_{3}, \alpha_{4}=\alpha_{5}$ and $\sum_{i=1}^{5} \alpha_{i}(t)<1$ for each $t>0$. It was proved that $S, T$ have a unique common fixed point if (1) is satisfied with $a_{i}$ replaced by $\alpha_{i}(d(x, y))$. By extending the idea of Boyd and Wong [5], we [31] introduced self-mappings

This research was supported by the National Research Council of Canada and by a grant from the Canadian Mathematical Congress. 
$\alpha_{i}, i=1,2, \cdots, 5$, on $[0, \infty)$ such that $\alpha_{2}=\alpha_{3}, \alpha_{4}=\alpha_{5}, \quad \sum_{i=1}^{5}\left(\alpha_{i}(t)<t\right.$ for all $t>0$ and each $\alpha_{i}$ is upper semicontinuous. It is assumed that for any distinct $x, y$ in $X,(1)$ is satisfied with $a_{i}$ replaced by $\alpha_{i}(d(x, y)) / d(x, y)$. We proved that either $S$ or $T$ has a fixed point and if both $S$ and $T$ have fixed points, then each of $S, T$ has a unique fixed point and these two fixed points coincide. Thus when $S=T, T$ has a unique fixed point. In fact, in this case, the condition "each $\alpha_{i}$ is upper semicontinuous" can be weakened to "each $\alpha_{i}$ is upper semicontinuous from the right". The conclusion of the above result is best possible in the sense that there are examples [30] of $S, T$ which satisfy the above conditions but $S$ has two fixed points and $T$ has none. However, in applying the above results, it may be difficult to find the required $\alpha_{i}$ 's even if they exist. We shall obtain some fixed point theorems by replacing each $a_{i}$ in (1) with a number $\alpha_{i}(x, y)$ depending on $\{x, y\}$, i.e. each $\alpha_{i}$ is a symmetric function of $X \times X$ into $[0, \infty)$. Thus each $\alpha_{i}$ need not be a composite function of $d$ with any function on the real line and it is possible that

$$
\sum_{i=1}^{5} \sup \left\{\alpha_{i}(x, y): x, y \in X\right\}>1 .
$$

One such example is given in Section 2. Related results are obtained for mappings in a Banach space.

THEOREM 1. Let $S, T$ be self-mappings on a complete metric space. Suppose that there exist functions $\alpha_{i}, i=1,2, \cdots, 5$, of $X \times X$ into $[0, \infty)$ such that

(a) $r \equiv \sup \left\{\sum_{i=1}^{5} \alpha_{i}(x y): x, y \in X\right\}<1$;

(b) $\alpha_{2}=\alpha_{3}, \alpha_{4}=\alpha_{5}$;

(c) for any distinct $x, y$ in $X$,

$$
\begin{aligned}
d(S(x), T(y)) \leqq & a_{1} d(x, y)+a_{2} d(x, T(y))+a_{3} d(y, S(x)) \\
& +a_{4} d(x, S(x))+a_{5} d(y, T(y)),
\end{aligned}
$$

where $a_{i}=\alpha_{i}(x, y)$.

Then $S$ or $T$ has a fixed point. If both $S$ and $T$ have fixed points, then each of $S, T$ has a unique fixed point and these two fixed points coincide.

PRoOF. Let $x_{0} \in X$,

$$
x_{2 n+1}=S\left(x_{2 n}\right), x_{2 n+2}=T\left(x_{2 n+1}\right), n=0,1,2, \cdots .
$$

We shall prove that $S$ or $T$ has a fixed point. For this purpose, we may assume that $x_{n} \neq x_{n+1}$ for each $n$. From (c) with $a_{i}=\alpha_{i}\left(x_{0}, x_{1}\right)$,

$$
d\left(x_{1}, x_{2}\right)=d\left(S\left(x_{0}\right), T\left(x_{1}\right)\right) \leqq\left(a_{1}+a_{4}\right) d\left(x_{0}, x_{1}\right)+a_{2} d\left(x_{0}, x_{2}\right)+a_{5} d\left(x_{1}, x_{2}\right) .
$$

Since $d\left(x_{0}, x_{2}\right) \leqq d\left(x_{0}, x_{1}\right)+d\left(x_{1}, x_{2}\right)$,

$$
\left(1-a_{2}-a_{5}\right) d\left(x_{1}, x_{2}\right) \leqq\left(a_{1}+a_{2}+a_{4}\right) d\left(x_{0}, x_{1}\right) \text {. }
$$


From (a) and (b), $a_{2}+a_{4} \leqq r / 2<1 / 2$ and

$$
\frac{a_{1}+a_{2}+a_{4}}{1-a_{2}-a_{5}} \leqq \frac{r-a_{2}-a_{4}}{1-a_{2}-a_{4}} \leqq \max \left\{\frac{r-x}{1-x}: x \in[0,1 / 2]\right\} \leqq r .
$$

From (3) and (4),

By induction, we have

$$
d\left(x_{1}, x_{2}\right) \leqq r d\left(x_{1}, x_{0}\right)
$$

$$
d\left(x_{n+2}, x_{n+1}\right) \leqq r d\left(x_{n+1}, x_{n}\right), \quad n=0,1,2, \cdots .
$$

By (5) and induction,

Since $r<1$,

$$
d\left(x_{n+1}, x_{n}\right) \leqq r^{n} d\left(x_{1}, x_{0}\right), \quad n=0,1,2, \cdots .
$$

$$
\sum_{n=0}^{\infty} d\left(x_{n+1}, x_{n}\right)<\infty
$$

and therefore $\left\{x_{n}\right\}$ is Cauchy. By completeness of $(X, d),\left\{x_{n}\right\}$ converges to some point $x$ in $X$. Since $x_{n+1} \neq x_{n}$ for each $n$, either $x_{2 n+1} \neq x$ for infinitely many $n$ or $x_{2 n} \neq x$ for infinitely many $n$. By symmetry, we may assume that $x_{2 n+1} \neq x$ for infinitely many $n$. Thus there is a subsequence $\{k(n)\}$ of $\{n\}$ such that $x_{2 k(n)+1} \neq x$ for each $n$. Let $n \geqq 1$. Then

$$
\begin{aligned}
d(x, T(x)) & \leqq d\left(x, x_{2 k(n)+1}\right)+d\left(x_{2 k(n)+1}, T(x)\right) \\
& =d\left(x, x_{2 k(n)+1}\right)+d\left(S\left(x_{2 k(n)}\right), T(x)\right) .
\end{aligned}
$$

From (c) with $a_{i}=\alpha_{i}\left(x_{2 k(n)}, x\right)$,

$$
\begin{aligned}
d\left(S\left(x_{2 k(n)}\right), T(x)\right) \leqq & a_{1} d\left(x_{2 k(n)}, x\right)+a_{2} d\left(x_{2 k(n)}, T(x)\right) \\
& +a_{3} d\left(x, x_{2 k(n)+1}\right) \\
& +a_{4} d\left(x_{2 k(n)}, x_{2 k(n)+1}\right)+a_{5} d(x, T(x)) \\
\leqq & d\left(x_{2 k(n)}, x\right)+\frac{r}{2} d\left(x_{2 k(n)}, T(x)\right) \\
& +d\left(x, x_{2 k(n)+1}\right) \\
& +d\left(x_{2 k(n)}, x_{2 k(n)+1}\right)+\frac{r}{2} d(x, T(x)) .
\end{aligned}
$$

From (6) and (7), we have by letting $n \rightarrow \infty$,

$$
d(x, T(x)) \leqq r d(x, T(x)) .
$$

Since $r<1, T(x)=x$. Let $x$ be a fixed point of $S$ and let $y$ be a fixed point of $T$. We need to prove that $x=y$. Suppose not. From (c) with $a_{i}=\alpha_{i}(x, y)$,

$$
d(x, y)=d(S(x), T(y)) \leqq\left(a_{1}+a_{2}+a_{3}\right) d(x, y)<d(x, y),
$$

a contradiction. 
THEOREM 2. Let $T$ be a self-mapping on a complete metric space $(X, d)$. Suppose that there exist symmetric functions $\alpha_{i}, i=1,2, \cdots, 5$, of $X \times X$ into $[0,1)$ such that

(a) $r \equiv \sup \left\{\sum_{i=1}^{5} \alpha_{i}(x, y): x, y \in X\right\}<1$;

(b) for any $x, y$ in $X$,

$$
\begin{aligned}
d(T(x), T(y)) \leqq & a_{1} d(x, y)+a_{2} d(x, T(y))+a_{3} d(y, T(x)) \\
& +a_{4} d(x, T(x))+a_{5} d(y, T(y)),
\end{aligned}
$$

where $a_{i}=\alpha_{i}(x, y)$.

Then $T$ has a unique fixed point.

Proof. Let $x, y \in X$. Calculating

$$
(d(T(x), T(y))+d(T(y), T(x))) / 2
$$

by (b), we obtain

where

$$
\begin{aligned}
d(T(x), T(y)) \leqq & b_{1} d(x, y)+b_{2} d(x, T(y))+b_{3} d(y, T(x)) \\
& +b_{4} d(x, T(x))+b_{5} d(y, T(y)),
\end{aligned}
$$

$$
\begin{array}{ll}
b_{1}=\left(\alpha_{1}(x, y)+\alpha_{1}(y, x)\right) / 2, \\
b_{2}=\left(\alpha_{2}(x, y)+\alpha_{3}(y, x)\right) / 2, & b_{3}=\left(\alpha_{2}(y, x)+\alpha_{3}(x, y)\right) / 2, \\
b_{4}=\left(\alpha_{4}(x, y)+\alpha_{5}(y, x)\right) / 2, & b_{5}=\left(\alpha_{4}(y, x)+\alpha_{5}(x, y)\right) / 2 .
\end{array}
$$

Since each $\alpha_{i}$ is symmetric, $b_{2}=b_{3}, b_{4}=b_{5}$ and

$$
\sum_{i=1}^{5} b_{i}=\sum_{i=1}^{5} \alpha_{i}(x, y) \leqq r .
$$

So we may assume that $\alpha_{2}=\alpha_{3}$ and $\alpha_{4}=\alpha_{5}$. By Theorem 1, $T$ has a unique fixed point.

EXAmpLe. Let $X$ be the unit interval with the usual distance. Let $T$ be the self-mapping on $X$ defined by

$$
\begin{array}{ll}
T(x)=\frac{109}{60} x-\frac{3}{2} & \text { if } \quad x \in[10 / 11,1] \\
T(x)=\frac{1}{6} x & \text { if } \quad x \in[0,10 / 11) .
\end{array}
$$

Then $T$ is a continuous increasing self-mapping on $X$. For $x, y$ in $[0,10 / 11)$, we define

$$
\alpha_{1}(x, y)=1 / 6, \alpha_{2}(x, y)=\alpha_{3}(x, y)=\alpha_{4}(x, y)=\alpha_{5}(x, y)=0 .
$$


For $x, y$ in $X$ with $x$ or $y$ in $[10 / 11,1]$, we define

$$
\alpha_{1}(x, y)=\alpha_{2}(x, y)=\alpha_{3}(x, y)=0, \alpha_{4}(x, y)=\alpha_{5}(x, y)=19 / 41 .
$$

Then each $\alpha_{i}$ is a symmetric function of $X \times X$ into $[0, \infty)$ and $\sum_{i=1}^{5} \alpha_{i} \leqq 38 / 41$ $<1$. Suppose that $x, y \in[10 / 11,1]$.

Then

$$
d(T(x), T(y))=\frac{109}{60} d(x, y) \leqq \frac{109}{660}
$$

and

$$
\alpha_{4}(x, y) d(x, T(x))+\alpha_{5}(x, y) d(y, T(y))=\frac{19}{41}\left(3-\frac{49}{60} x-\frac{49}{60} y\right) \geqq \frac{418}{660} .
$$

So $T$ is not nonexpansive on $X$ and

$$
d(T(x), T(y)) \leqq \alpha_{4}(x, y) d(x, T(x))+\alpha_{5}(x, y) d(y, T(y)) .
$$

Now suppose that $x \in[10 / 11,1], y \in[0,10 / 11)$. Then

Since

$$
\begin{aligned}
& d(T(x), T(y))=T(x)-T(y)=\frac{109}{60} x-\frac{3}{2}-\frac{1}{6} y \\
& \alpha_{4}(x, y) d(x, T(x))+\alpha_{5}(x, y) d(y, T(y))=\frac{19}{41}\left(\frac{3}{2}-\frac{49}{60} x+\frac{5}{6} y\right) .
\end{aligned}
$$

$$
\frac{109}{60} x-\frac{3}{2}-\frac{1}{6} y-\frac{19}{41}\left(\frac{3}{2}-\frac{49}{60} x+\frac{5}{6} y\right)=\frac{90}{41}(x-1)-\frac{68}{123} y \leqq 0,
$$

(10) is satisfied. Finally, suppose that $x, y \in[0,10 / 11)$.

Then

$$
d(T(x), T(y))=\frac{1}{6} d(x, y)=\alpha_{5}(x, y) d(x, y) .
$$

So $T$ satisfies the conditions of Theorem 2. To see the advantage of Theorem 2, we note that

$$
\sum_{i=1}^{5} \sup \left\{\alpha_{i}(x, y): x, y \in X\right\}=\frac{269}{246}>1
$$

and $\alpha_{4}$ is not a composite function of $d$ with any function on the real line $\left(\alpha_{4}(0,1 / 11) \neq \alpha_{4}(10 / 11,1)\right.$ but $\left.d(0,1 / 11)=d(10 / 11,1)\right)$. Also, if we replace $1 / 6$ in (9) by a number in $[0,1 / 6)$, we will obtain an example of $T$ which is not continuous on $X$ and satisfies the conditions of Theorem 2.

Let $X$ be a complete metric space. For any subset $A$ of $X, \operatorname{cl} A$ will denote the closure of $A$ in $X$ and $\delta(A)$ will denote the diameter of $A$, i.e. $\delta(A)=\sup \{d(x, y)$ : 
$x, y \in A\}$. Let $T$ be a self-mapping on $X$. A subset $A$ of $X$ is $T$-invariant if $T(A) \subset A$. Let $x \in X$. $O(x)$ will denote the set $\left\{T^{n}(x): n \geqq 0\right\}\left(T^{0}(x)=x\right)$ of all iterates $T^{n}(x)$ of $x$ and will be called the orbit of $x$. T has orbital diminishing diameters if for any $x$ in $X$, either $\delta(O(x))=0$ or

$$
\lim _{n \rightarrow \infty} \delta\left(O\left(T^{n}(x)\right)<\delta(O(x))\right.
$$

This definition was introduced by Belluce and Kirk [3], [4], [18]. Kirk [18] proved that if $X$ is compact and if $T$ is a continuous self-mapping on $X$ which has diminishing orbital diameters, then for any $x$ in $X$, some subsequence of $\left\{T^{n}(x)\right\}$ converges to a fixed point of $T$. We shall obtain a related result with a different approach.

THEOREM 3. Let $T$ be a continuous self-mapping on a compact metric space. Suppose that

(a) there exist symmetric functions $\alpha_{1}, \alpha_{2}, \alpha_{3}$, of $X \times X$ into $[0,1]$ such that $\alpha_{1}+\alpha_{2}+\alpha_{3} \leqq 1$ and for any $x, y$ in $X$,

$$
d(T(x), T(y)) \leqq a_{1} d(x, y)+a_{2} d(x, T(y))+a_{3} d(y, T(x)),
$$

where $a_{i}=\alpha_{i}(x, y)$;

(b) for any T-invariant closed subset $A$ of $X$ with $\delta(A)>0$, there exist $y, z$ in $A$ such that

$$
\sup \left\{d\left(y, T^{n}(z)\right): n \geqq 0\right\}<\delta(A) .
$$

Then for any $x$ in $X,\left\{T^{n}(x)\right\}$ has a subsequence which converges to a fixed point of $T$.

Proof. Let $x \in X$. Consider $\operatorname{cl} O(x)$. Then $\mathrm{cl} O(x)$ is compact and $T$-invariant. By Zorn's lemma, $\operatorname{cl} O(x)$ includes a minimal non-empty closed $T$-invariant subset $Y$ of $X$. Suppose to the contrary that $\delta(Y)>0$. Then by (b), there exist $y, z$ in $Y$ such that

$$
r \equiv \sup \left\{d\left(y, T^{n}(z)\right): n \geqq 0\right\}<\delta(Y) .
$$

By continuity of $T, d(y, u) \leqq r$ for each $u \in \operatorname{cl} O(z)$. Since $\operatorname{cl} O(z)$ is $T$-invariant, by minimality of $Y, \operatorname{cl} O(z)=Y$. So the set

$$
W=\{u \in Y: d(u, v) \leqq r \text { for each } v \text { in } Y\}
$$

contains $y$. By continuity of $d, W$ is closed. Let $u \in W$. By compactness of $Y$, there exists $v_{0} \in Y$ such that

$$
d\left(T(u), v_{0}\right)=\sup \{d(T(u), v): v \in Y\} .
$$

Since $T$ is continuous, $T(Y)$ is compact. Also, $T(Y)$ is $T$-invariant. So by minimality of $Y, T(Y)=Y$. Therefore there exists $v_{1}$ in $Y$ such that $T\left(v_{1}\right)=v_{0}$. By (11), (12) and (a) with $a_{i}=\alpha_{i}\left(u, v_{1}\right)$, 


$$
\begin{aligned}
d\left(T(u), v_{0}\right) & =d\left(T(u), T\left(v_{1}\right)\right) \\
& \leqq a_{1} d\left(u, v_{1}\right)+a_{2} d\left(u, T\left(v_{1}\right)\right)+a_{3} d\left(v_{1}, T(u)\right) \\
& \leqq a_{1} r+a_{2} r+a_{3} d\left(T\left(u, v_{0}\right) \leqq\left(1-a_{3}\right) r+a_{3} d\left(T(u), v_{0}\right) .\right.
\end{aligned}
$$

So

$$
\left(1-a_{3}\right) d\left(T(u), v_{0}\right) \leqq\left(1-a_{3}\right) r .
$$

Since each $\alpha_{i}$ is symmetric, we may assume that $a_{2}=a_{3}$. Thus $a_{3} \leqq 1 / 2$. From (13), we have $d\left(T(u), v_{0}\right) \leqq r$. So $W$ is $T$-invariant. By minimality of $Y, W=Y$. Therefore by (11),

$$
\delta(Y)=\delta(W) \leqq r<\delta(Y),
$$

a contradiction. Hence $\delta(Y)=0$ and the point $x_{0}$ in $Y$ is a fixed point of $T$. Since $x_{0}$ is a fixed point of $T$ and $x_{0} \in \operatorname{cl} O(x)$, some subsequence of $\left\{T^{n}(x)\right\}$ converges to $x_{0}$.

We remark that in Theorem 3, (b) is satisfied if $T$ has diminishing orbital diameters.

Let $B$ be a Banach space. $d$ will denote the metric for $B$ induced by the norm \|\| of $B$. For any subset $A$ of $B, \operatorname{co} A$ will denote the convex hull of $A$. Let $X$ be a bounded closed convex subset of $B$. Let $T$ be a self-mapping on $X . X$ is regular with respect to $T$ if for any non-empty closed convex $T$-invariant subset $A$ of $X$, either $\delta(A)=0$ or there exist $y, z$ in $A$ such that

$$
\sup \left\{d\left(y, T^{n}(z)\right): n \geqq 0\right\}<\delta(A) .
$$

$X$ is normal with respect to $T$ if for any non-empty closed convex $T$-invariant subset $A$ of $X$, either $\delta(A)=0$ or there exists $z$ in $A$ such that

$$
\sup \{d(z, y): y \in A\}<\delta(A) .
$$

$X$ has normal structure [6] if $X$ is normal with respect to the identity function on $X$. It is clear that $X$ is regular with respect to $T$ if it is normal with respect to $T$; $X$ is normal with respect to $T$ if it has normal structure. Our notions of regularity and normality link $T$ with the convex structure of $X$.

THEOREM 4. Let $X$ be a weakly compact convex subset of a Banach space B. Let $T$ be a self-mapping on $X$. Suppose that

(a) $X$ is normal with respect to $T$;

(b) there exist symmetric functions $\alpha_{1}, \alpha_{2}, \alpha_{3}$ of $X \times X$ into $[0, \infty)$ such that $\alpha_{1}+\alpha_{2}+\alpha_{3} \leqq 1$ and for any $x, y$ in $X$,

$$
\left.\left.d(T(x), T(y)) \leqq a_{1} d(x, y)+a_{2} d(x, T) y\right)\right)+a_{3} d(y, T(x)),
$$

where $a_{i}=\alpha_{i}(x, y)$.

Then $T$ has a fixed point. 
Proof. By Zorn's lemma, there exists a minimal non-empty closed convex $T$-invariant subset $Y$ of $X$. Suppose to the contrary that $\delta(Y)>0$. Then by (a), there exists $z$ in $Y$ such that

So

$$
r \equiv \sup \{d(z, y): y \in Y\}<\delta(Y) .
$$

$$
W=\{w \in Y: d(w, y) \leqq r \text { for each } y \text { in } Y\}
$$

contains $z$. Obviously $W$ is convex and closed. We shall prove that $W$ is $T$ invariant. Let $w \in W$,

$$
r_{1}=\sup \{d(T(w), x): x \in Y\} .
$$

Since $T(Y) \subset Y$ and $Y$ is closed and convex, one has cl co $T(Y) \subset Y$; hence

$$
T(\mathrm{cl} \operatorname{co} T(Y)) \subset T(Y) \subset \operatorname{cl} \operatorname{co} T(Y) .
$$

By minimality of $Y, \operatorname{cl} \operatorname{co} T(Y)=Y$. So by continuity and convexity of $d$,

$$
r_{1}=\sup \{d(T(w), T(y)): y \in Y\} .
$$

(For nonexpansive mappings, the above argument or its variants occurred in [1], [2], [3], [14], [16], [17], [19], [20], [21], [22], [23], [28], [29].) Let $w \in W$, $y \in Y$. By (b) with $a_{i}=\alpha_{i}(x, y)$,

$$
d(T(w), T(y)) \leqq a_{1} d(w, y)+a_{2} d(w, T(y))+a_{3} d(y, T(w)) \leqq a_{1} r+a_{2} r+a_{3} r_{1} .
$$

Let $\varepsilon>0$ and select $\bar{y} \in Y$ so that

$$
\sup _{y \in Y}\left(a_{1} r+a_{2} r+a_{3} r_{1}\right) \leqq \bar{a}_{1} r+\bar{a}_{2} r+\bar{a}_{3} r_{1}+\varepsilon \text { where } \bar{a}_{i}=\alpha_{i}(w, \bar{y}) .
$$

Then

$$
\begin{aligned}
r_{1}=\sup _{y \in Y} d(T(w), T(y)) & \leqq \sup _{y \in Y}\left(a_{1} r+a_{2} r+a_{3} r_{1}\right) \leqq \tilde{a}_{1} r+\tilde{a}_{2} r+\tilde{a}_{3} r_{1}+\varepsilon \\
& =\left(1-\bar{a}_{3}\right) r+\tilde{a}_{3} r_{1}+\varepsilon .
\end{aligned}
$$

Hence, since $\bar{a}_{3} \leqq \frac{1}{2}$,

$$
r_{1} \leqq r+\frac{\varepsilon}{1-\tilde{a}_{3}} \leqq r+2 \varepsilon .
$$

Since $\varepsilon$ is arbitrary, $r_{1} \leqq r$. A contradiction can be obtained as in the proof of Theorem 3. Hence $Y$ is a singleton and the point in $Y$ is a fixed point of $T$.

By refining the above argument, one can obtain the following result for nonexpansive mappings.

THEOREM 5. Let $X$ be a weakly compact convex subset of a Banach space. Let $T$ be a nonexpansive self-mapping on $X$. Suppose that $X$ is regular with respect to $T$. Then $T$ has a fixed point. 
Let $X$ be a weakly compact convex subset of a Banach space $B$. Let $T$ be a nonexpansive self-mapping on $X$. Browder [7] and Göhde [12] proved that $T$ has a fixed point if $B$ is uniformly convex. Belluce and Kirk [1] proved that $T$ has a fixed point if $X$ has normal structure. Then they [3] obtained the following more general results: (i) $T$ has a fixed point if for any $x$ in $X$, cl co $O(x)$ has normal structure, (ii) $T$ has a fixed point if $T$ has diminishing orbital diameters. Theorem 5 combines all these results into a more general one.

We remark here that by modifying the definitions, conditions and proofs in an obvious way, Theorem 3 can be proved for a compact Hausdorff topological space $X$ associated with a definite family of pseudo-metrics; Theorems 4 and 5 can be proved for a locally convex Hausdorff topological vector space $X$ associated with a family of pseudo-norms. One such example can be found in [14] Also, every uniformly convex Banach space is reflexive [24]. So in this case, to assiume that $X$ is weakly compact convex is the same as assuming that $X$ is bounded, closed and convex.

Let $X$ be a bounded convex subset of a Banach space $B$. Let $y \in X$,

$$
\begin{aligned}
& r_{y}=\sup \{d(x, y): x \in X\}, \\
& r=\inf \left\{r_{y}: y \in X\right\}, \\
& C=\left\{x \in X: r_{x}=r\right\} .
\end{aligned}
$$

$r$ is called the radius of $X$ and $C$ is called the Chebyshev centre of $X$. It was shown in [17] that if $B$ is reflexive and $X$ is weakly compact convex, then $C$ is a non-empty closed convex subset of $X$. If $C$ is a singleton $\{x\}$, then $x$ is called the generalized center of $X$. For example, every bounded closed convex subset of a uniformly convex Banach space has a generalized centre [11].

THEOREM 6. Let $X$ be a bounded convex subset of a Banach space B. Suppose that $X$ has a generalized centre $x_{0}$. Let $T$ be a self-mapping on $X$ (not necessarily continuous). Suppose further that

(a) $\operatorname{cl} \operatorname{co} T(X) \supset X$;

(b) there exist symmetric functions $\alpha_{1}, \alpha_{2}, \alpha_{3}$ of $X \times X$ into $[0, \infty)$ such that for all $x, y$ in $X$,

$$
d(T(x), T(y)) \leqq a_{1} d(x, y)+a_{2} d(x, T(y))+a_{3} d(y, T(x)),
$$

where $a_{i}=\alpha_{i}(x, y)$.

Then $x_{0}$ is a fixed point of $T$.

ProOF. Form the definition of generalized centres, 


$$
\left\{x_{0}\right\}=\bigcap_{y \in Y}\{x \in Y: d(x, y) \leqq r\},
$$

where $r$ is the radius of $Y$. Let $x \in Y$. By (b) with $a_{i}=\alpha_{i}\left(x_{0}, x\right)$,

$$
\begin{aligned}
d\left(T\left(x_{0}\right), T(x)\right) & \leqq a_{1} d\left(x_{0}, x\right)+a_{2} d\left(x_{0}, T(x)\right)+a_{3} d\left(x, T\left(x_{0}\right)\right) \\
& \leqq a_{1} r+a_{2} r+a_{3} r_{1},
\end{aligned}
$$

where $r_{1}=\sup \left\{d\left(T\left(x_{0}\right), y\right): y \in Y\right\}$. By (a),

$$
r_{1}=\sup \left\{d\left(T\left(x_{0}\right), T(y)\right): y \in Y\right\} .
$$

Arguing as the the proof of Theorem $4, r_{1} \leqq r$. So

$$
T\left(x_{0}\right) \in \bigcap_{y \in Y}\{x \in Y: d(x, y) \leqq r\} .
$$

So by (14), $T\left(x_{0}\right)=x_{0}$.

Let $X$ be a weakly compact convex subset of a Banach space $B$. Let $T$ be a self-mapping on $X . T$ is called a generalized nonexpansive mapping if there exist symmetric functions $\alpha_{i}, i=1,2, \cdots, 5$, of $X \times X$ into $X$ such that $\sum_{i=1}^{5} \alpha_{i} \leqq 1$ and for all $x, y$ in $X$,

$$
\begin{aligned}
d(T(x), T(y)) \leqq & a_{1} d(x, y)+a_{2} d(x, T(y))+a_{3} d(y, T(x)) \\
& a_{4} d(x, T(x))+a_{5} d(y, T(y)),
\end{aligned}
$$

where $a_{i}=\alpha_{i}(x, y)$. Let $T$ be a generalized nonexpansive self-mapping on $X$. We post the following open questions:

(a) $\inf \{d(x, T(x)): x \in X\}=0$ ?

(b) Does $T$ have a fixed point?

By using the asymptotic center recently introduced by Edelstein [10], we can prove that if $X$ is uniformly convex and if $T$ is continuous, then the above questions are equivalent. It is also interesting to note that if $T$ has a fixed point, then $T$ must be quasi-nonexpansive [9].

We would like to mention here that the use of five monotonically nonincreasing functions $\alpha_{i}$ on $(0, \infty)$ (with $\left.\sum_{i=1}^{5} \alpha_{i}<1\right)$ to contract self-mappings on a complete metric space is invented by Hardy and Rogers [13].

\section{References}

[1] L. P. Belluce and W. A. Kirk, 'Fixed-point theorems for families of contraction mappings', Pacific J. Math. 18 (1966), 213-217.

[2] L. P. Belluce and W. A. Kirk, 'Nonexpansive mappings and fixed-points in Banach spaces', Illinois J. Math. 11 (1967), 471-479.

[3] L. P. Belluce and W. A. Kirk, 'Fixed point theorems for certain classes of nonexpansive mappings', Proc. Amer. Math. Soc. 20 (1969), 141-146. 
[4] L. P. Belluce and W. A. Kirk, 'Some fixed point theorems in a metric and Banach space', Canad. Math. Bull. 12 (1969), $481-489$.

[5] D. W. Boyd and J. S. W. Wong, 'On nonlinear contractions', Proc. Amer. Math. Soc. 20 (1969), 458-464.

[6] M. S. Brodskii and D. P. Milman, 'On the center of a convex set', Dokl. Akad. Nauk. SSSR (N.S.) 59 (1948), 837-840.

[7] F. E. Browder, 'Nonexpansive nonlinear operators in a Banach space', Proc. Nat. Acad. Sci. U.S.A. (1966), 1041-1044.

[8] J. A. Clarkson, 'Uniformly convex spaces', Trans. Amer. Math. Soc. 40 (1936), 396-414.

[9] W. G. Dotson, Jr., 'Fixed points of quasi-nonexpansive mappings', J. Austral. Math. Soc. 13 (1972), 167-170.

[10] M. Edelstein, 'The construction of an asymptotic center with a fixed point property', Bull. Amer. Math. Soc. 18 (1972), 206-208.

[11] Klaus Floret, 'Eine Bermekung über a priori-Fixpunkte nicht-expansiver Abbildungen', Manuscripta math. 6 (1972), 321-326.

[12] Von Dretrich Göhde, 'Zum prinzip der Konstraktiven Abbildung', Math. Nachr. 30 (1965), 251-258.

[13] G. Hardy and T. Rogers, 'A generalization of a fixed point theorem of Reich', Canad. Math. Bull. 16 (1973), 201-206.

[14] R. D. Holmes and Anthony T. Lau, 'Non-expansive actions of topological semigroups' (to appear in J. London Math. Soc.).

[15] R. Kannan. 'Some results on fixed points II', Amer. Math. Monthly 76 (1969), 405-408.

[16] Yōichi Kijima and Wataru Takahashi, 'A fixed point theorem for nonexpansive mappings in metric space', Kodai Math. Sem. Rep. 21 (1969), 326-330.

[17] W. A. Kirk, 'A fixed point theorem for mappings which do not increase distance', Amer. Math. Monthly 72 (1965), 1004-1007.

[18] W. A. Kirk, 'On mappings with diminishing orbital diameters', J. London Math. Soc. 44 (1969), 107-111.

[19] W. A. Kirk, 'Fixed point theorems for nonexpansive mappings', Proc. Sympos. Pure Math. 18, Amer. Math. Soc., Providence, R.I. (1970), 162-168.

[20] W. A. Kirk and W. D. Royalty, 'Fixed point theorems for certain nonexpansive mappings', Illinois J. Math. 15 (1971), 656-663.

[21] Anthony To-Ming Lau, 'Invariant means on almost periodic functions and fixed point properties', (to appear in Rocky Mountain J. Math.).

[22] Ralph De Marr, 'Common fixed-points for commuting contraction mappings', Pacific J. Math. 13 (1963), 1139-1141.

[23] Theodore Mitchell, 'Fixed points of reversible semigroups of nonexpansive mappings', Kodai Math. Sem. Rep. 22 (1970), 322-323.

[24] B. J. Pettis, 'A proof that every uniformly convex space is reflexive', Duke Math.J. 5 (1939), 249-253.

[25] E. A. Rakotch, 'A note on contractive mappings', Proc. Amer. Math. Soc. 13 (1962), 459-465.

[26] Simon Reich, 'Some remarks concerning contraction mappings', Canad. Math. Bull. 14 (1971), 121-124.

[27] Pramila Srivastava and Vijay Kummer Gupta, 'A note on common fixed points', Yokohama Math.J. 19 (1971), 91-95.

[28] Wataru Takahashi, 'Fixed point theorem for amenable semigroups of nonexpansive mappings', Kodai Math. Sem. Rep. 21 (1969), 383-386. 
[29] Wataru Takahashi, 'A convexity in metric space and nonexpansive mappings', I, Kodai Math. Sem. Rep. 22 (1970), 142-149.

[30] Chi Song Wong, 'Common fixed points of two mappings', Pacific J. Math. 48 (1973), 299-312.

[31] Chi Song Wong, 'Generalized contractions and fixed point theorems', Proc. Amer. Math. Soc. 42 (1974), 409-417.

Summer Research Institute

Department of Mathematics

University of Alberta

Edmonton, Alberta

Canada

Present address:

University of Windsor

Windsor, Ontario

Canada 